\title{
SOME EXACT SOLUTIONS OF CERTAIN FIELD EQUATIONS IN GENERAL RELATIVITY
}

\author{
L. K. PATEL \\ (Received 26 April 1974; revised 1 July 1974) \\ Communicated by C. A. Hurst
}

\begin{abstract}
Summary
A general metric is considered. Some solutions corresponding to the field equations $R_{i k}-\lambda g_{i k}=$ $-8 \pi E_{i k}$ are obtained as particular cases. Details of these solutions are also discussed. An Einstein space corresponding to the field equations $R_{i k}=\lambda g_{i k}$ is also constructed as a particular case of the general metric.
\end{abstract}

\section{Introduction}

An electrovac universe with a non-zero cosmological constant has been obtained by Patel and Vaidya (1971) as an exact solution of the field equations

$$
R_{i k}-\lambda g_{i k}=-8 \pi E_{i k}
$$

where $R_{i k}$ are the components of Ricci tensor and $E_{i k}$ are components of electromagnetic energy tensor.

In Section 3 of the present paper we shall discuss another solution of the field equations (1). In Section 4 we shall construct an Einstein space.

For the description of our solutions we require some geometrical preliminaries developed by Patel and Vaidya (1969). So we begin with a brief outline of these preliminaries.

In Minkowskian space-time we can find four uniform vector fields such that (i) any two of them are mutually orthogonal and (ii) one of them is time-like and the other three space-like. Let $\lambda^{i}$ be the unit tangent to the time-like vector through a point $P$ (co-ordinates $x^{i}$ ) and $A^{i}, B^{i}, C^{i}$ be the unit tangents to the space-like vectors through $P$. We use the signature $(-,-,-,+)$ and raise and lower indices with the help of the Minkowskian metric tensor $\eta_{i k}$ or $\eta^{i k}$. These four uniform vector fields give rise to a Euclidean reference frame with coordinates $x, y, z, t$ for $P$ where $x=x^{i} A_{i}, y=x^{i} B_{i}, z=x^{i} C_{i}, t=x^{i} \lambda_{i}$ so that 
$x_{, k}=A_{k}, \quad y_{, k}=B_{k}, \quad z_{, k}=C_{k}$ and $t_{, k}=\lambda_{k}$, a comma indicating an ordinary derivative.

\section{A General Metric}

Consider a Riemannian 4-space described by the metric

$$
d s^{2}=g_{i k} d x^{i} d x^{k}
$$

where

$$
g_{i k}=\eta_{i k}+M B_{i} B_{k}+N C_{i} C_{k}
$$

Here $M$ is a function of $x$ only and $N$ is a function of $t$ only.

The contravariant components $g^{i k}$ and the determinant $g$ of the metric tensor $g_{i k}$ are given by

$$
g^{i k}=\eta^{i k}-\frac{M}{1-M} B^{i} B^{k}-\frac{N}{1-N} C^{i} C^{k}, g=-(1-M)(1-N)
$$

As $g$ is negative $(1-M)$ and $(1-N)$ should be of the same sign. From (4) it is easy to see that

$$
g^{i k} B_{i} B_{k}=-\frac{1}{1-M} \text { and } g^{i k} C_{i} C_{k}=-\frac{1}{1-N}
$$

$B_{i}$ and $C_{i}$ are space-like unit vectors with respect to the Minkowskian metric. In order to have the spacelike character of $B_{i}$ and $C_{i}$ with respect to the metric (2), it is essential that $1-M>0,1-N>0$. The 3 -index symbols for the metric (2) are given by

$$
\begin{aligned}
\Gamma_{i k}^{n}= & \frac{M^{\prime}}{2(1-M)} B^{n}\left(A_{k} B_{i}+A_{i} B_{k}\right)-\frac{M^{\prime}}{2} A^{n} B_{i} B_{k} \\
& +\frac{\dot{N} C^{n}}{2(1-N)}\left(\lambda_{k} C_{i}+\lambda_{i} C_{k}\right)-\frac{\dot{N}}{2} \lambda^{n} C_{i} C_{k}
\end{aligned}
$$

Here and in what follows an overhead dash denotes the differentiation with respect to $x$ and an overhead dot denotes the differentiation with respect to $t$.

The Ricci-tensor $R_{i k}$ for the metric (2) can be expressed as

$$
R_{i k}=f f^{\prime \prime}\left\{B_{i} B_{k}+\frac{1}{f^{2}} A_{i} A_{k}\right\}-F \ddot{F}\left\{C_{i} C_{k}-\frac{1}{F^{2}} \lambda_{i} \lambda_{k}\right\}
$$

where $1-M=f^{2}$ and $1-N=F^{2}$.

\section{Electrovac Universes}

Case (i). Let us choose the electromagnetic four potential $\phi_{i}$ as

$$
\phi_{i}=\alpha B_{i}+\beta C_{i},
$$


where $\alpha$ is a function of $x$ and $\beta$ is a function of $t$ only. The Maxwell equation $F_{: k}^{i k}=0$ becomes

$$
\frac{F}{f^{2}}\left(f \alpha^{\prime \prime}-\alpha^{\prime} f^{\prime}\right) B^{i}+\frac{f}{F^{2}}(F \ddot{\beta}-\dot{\beta} \dot{F}) C^{i}=0 .
$$

From this equation we have $\alpha=f k$ and $\dot{\beta}=F l$ where $k$ and $l$ are integration constants.

The electromagnetic energy tensor $E_{i k}$ for the choice (8) of $\phi_{i}$ can be expressed as

$$
E_{i k}=\frac{1}{2}\left(k^{2}+l^{2}\right)\left[f^{2} B_{i} B_{k}+A_{i} A_{k}+\lambda_{i} \lambda_{k}-F^{2} C_{i} C_{k}\right]
$$

The Einstein-Maxwell equations $R_{i k}-\lambda g_{i k}=-8 \pi E_{i k}$ can be expressed in the explicit form as

$$
\begin{gathered}
\left(\frac{f^{\prime \prime}}{f}+\lambda\right)\left(A_{i} A_{k}+f^{2} B_{i} B_{k}\right)+\left(\frac{\ddot{F}}{F}-\lambda\right)\left(\lambda_{i} \lambda_{k}-F^{2} C_{i} C_{k}\right) \\
=-a^{2}\left(A_{i} A_{k}+f^{2} B_{i} B_{k}+\lambda_{i} \lambda_{k}-F^{2} C_{i} C_{k}\right)
\end{gathered}
$$

where $\lambda$ is a cosmological constant and $a^{2}=4 \pi\left(k^{2}+l^{2}\right)$. These equations imply that

$$
f^{\prime \prime}=-\left(\lambda+a^{2}\right) f \quad \text { and } \quad \ddot{F}=\left(\lambda-a^{2}\right) F
$$

The solutions of the above differential equations depend upon the nature of the constant $\lambda$. Here we have to consider the following possibilities:

$$
\begin{aligned}
& \text { (i) } \lambda=0 \text { (ii) } 0<\lambda<a^{2} \text { (iii) } \lambda=a^{2} \text { (iv) } \lambda>a^{2} \\
& \text { (v) }-a^{2}<\lambda<0 \text { (vi) } \lambda=-a^{2} \text { (vii) } \lambda<-a^{2}
\end{aligned}
$$

For brevity we shall write the solution corresponding to the possibility (i) only in the explicit form. The explicit forms of the solutions corresponding to the other possibilities can be written down on similar lines.

If $\lambda=0$, we obtain

$$
\begin{aligned}
& f=A \sin a x+B \cos a x \\
& F=A_{1} \sin a t+B_{1} \cos a t
\end{aligned}
$$

and

$$
\alpha=\frac{k}{a}(-A \cos a x+B \sin a x)+m
$$

$$
\beta=\frac{k}{a}(-A \cos a t+B \sin a t)+n
$$

where $m, n, A, B, A_{1}, B_{1}$ are integration constants. 
The metric for the above solution can be expressed as

$$
\begin{aligned}
d s^{2}= & d t^{2}-d x^{2}-(A \sin a x+B \cos a x)^{2} d y^{2} \\
& -\left(A_{1} \sin a t+B_{1} \cos a t\right)^{2} d z^{2}
\end{aligned}
$$

The above mentioned solution is discussed by Pandey (1962). Here it should be noted that when $k=l$ we get a null electromagnetic field.

Case (ii). Let us take $N=0$ and choose the electromagnetic four potential

$$
\phi_{i} \quad \text { as } \quad \phi_{i}=\alpha B_{i}
$$

where $\alpha$ is a function of $x$ only.

Then the Einstein-Maxwell field equations (1) and $F^{i k} ; k=0$ imply

$$
f=B \cos (\sqrt{2 \lambda} x+c), \lambda=A^{2}, \alpha=-\frac{B}{2} \sin (\sqrt{2 \lambda} x+c)+l
$$

where $B, C$ and $l$ are constants. This solution is nothing but the solution describing a simple magnetic universe discussed by Patel and Vaidya (1971).

Case (iii). Let us take $M=0$ and choose the electromagnetic four potential $\phi_{i}$ as

$$
\phi_{i}=\beta C_{i}
$$

where $\beta$ is a function of $t$ only.

Then Einstein-Maxwell equations (1) and

$$
F^{i k} ; k=0 \text { imply }
$$

$$
F=B \cos \sqrt{-2 \lambda} t+C), \lambda=-A^{2}, \beta=\frac{B}{2} \sin \sqrt{-2 \lambda} t+l
$$

where $B, C$ and $l$ are constants.

By a co-ordinate transformation one can make $B=1$ and $C=0$. Therefore the explicit form of the line-element for the above solution is

$$
d s^{2}=d t^{2}-d x^{2}-d y^{2}-\cos ^{2}(\sqrt{-2 \lambda} t) d z^{2}
$$

The line-element (16) is plane symmetric as it obviously admits of the group of motions.

$$
\bar{x}=x+a, \bar{y}=y+b
$$

and also rotation above $z$-axis. Further it admits the motion $\bar{z}=z+c$. Here $a, b, c$ are constants. Thus it admits of a four parameter group of motions.

The Riemannian 4-space described by the metric (16) can be embeded in a five-dimensional pseudo-Euclidean space. The metric (16) can be expressed as 


$$
d s=\left(d z^{1}\right)^{2}-\left(d z^{2}\right)^{2}-\left(d z^{3}\right)^{2}-\left(d z^{4}\right)^{2}-\left(d z^{5}\right)^{2}
$$

where

$$
\begin{aligned}
& z^{1}=t-\lambda t+\sqrt{\frac{-\lambda}{8}} \sin \sqrt{-8 \lambda} t, z^{2}=x, z^{3}=y, \\
& z^{4}=\sin z \cos \sqrt{-2 \lambda} t, z^{5}=\cos z \cos \sqrt{-2 \lambda} t .
\end{aligned}
$$

Thus the Riemannian 4-space describing by the metric (16) is of class one.

The cosmological constant $\lambda$ plays an important role in our solution. If we set $\lambda=0$, the electromagnetic field disappears and the geometry of the universe becomes Minkowskian. Thus in this respect the above solution behaves like the simple magnetic universe discussed by Patel and Vaidya (1971).

\section{An Einstein Space}

In this section we shall consider the space-time given by the metric (2).

Now, for an Einstein space we must have

$$
R_{i k}=\lambda g_{i k}
$$

where $\lambda$ is a constant.

We also know that

$$
\eta_{i k}=\lambda_{i} \lambda_{k}-A_{i} A_{k}-B_{i} B_{k}-C_{i} C_{k}
$$

Therefore

$$
g_{i k}=\lambda_{i} \lambda_{k}-A_{i} A_{k}-f^{2} B_{i} B_{k}-F^{2} C_{i} C_{k}
$$

The results (18), (20) and (7) imply that

$$
\ddot{F}=\lambda F \quad \text { and } \quad \ddot{f}=-\lambda f
$$

If $\lambda$ is positive, say $a^{2}$, then

$$
f=A_{1} \cos \left(a x+B_{1}\right), F=A e^{a t}+B e^{-a t}
$$

If $\lambda$ is negative, say $-b^{2}$, then

$$
f=A_{1} e^{b x}+B_{1} e^{-b x}, F=A \cos (b t+B)
$$

In (22) and (23), $A, A_{1}, B$ and $B_{1}$ are constants of integration. The explicit forms of the line-element for positive and negative values of $\lambda$ are

$$
d s^{2}=d t^{2}-d x^{2}-A_{1}^{2} \cos ^{2}\left(a x+B_{1}\right) d y^{2}-\left(A e^{a t}+B e^{-a t}\right) d z^{2}
$$




$$
d s^{2}=d t^{2}-d x^{2}-\left(A_{1} e^{b x}+B_{1} e^{-b x}\right)^{2} d y^{2}-A^{2} \cos ^{2}(b t+B) d z^{2}
$$

respectively.

It is clear that when $\lambda=0$ the space-time becomes flat. This is a peculiar feature of the Einstein space discussed above.

\section{Acknowledgments}

The author wishes to thank Professor P. C. Vaidya and Dr. M. Misra for many helpful discussions. His thanks are also due to the referee for helpful comments.

\section{References}

L. K. Patel and P. C. Vaidya (1971), 'A simple magnetic universe', Curr. Sci. 40, 288. 158-164.

L. K. Patel and P. C. Vaidya (1969), 'On plane symmetric cosmological models', Progress of Mathematics 3, 158.

S. N. Pandey (1962), 'Photon Fluid Models in Relativistic cosmology', Proc. Nat. Inst. India 28, 646-654.

Department of Mathematics

Gujarat University

Ahmedabad 380009

India. 